\title{
The tubovesicular network in Plasmodium vivax liver-stage hypnozoites and schizonts associates with host aquaporin 3
}

Authors: Kayla Sylvester, ${ }^{1}$ Steven P. Maher, ${ }^{2}$ Dora Posfai, ${ }^{3}$ Michael K. Tran, ${ }^{3}$ McKenna C. Crawford, ${ }^{3}$ Amélie Vantaux, ${ }^{4}$ Benoît Witkowski, ${ }^{4}$ Dennis E. Kyle,${ }^{2}$ and Emily R. Derbyshire ${ }^{1,3}$

\author{
Affiliations \\ ${ }^{1}$ Department of Molecular Genetics and Microbiology, Duke University Medical Center, Durham, \\ North Carolina, 27707, United States of America \\ ${ }^{2}$ Center for Tropical and Emerging Global Diseases, University of Georgia, Athens, Georgia, \\ 30602, United States of America \\ ${ }^{3}$ Chemistry Department, Duke University, Durham, North Carolina, 27707, United States of \\ America \\ ${ }^{4}$ Malaria Molecular Epidemiology Unit, Pasteur Institute in Cambodia, Phnom Penh, 12201, \\ Cambodia
}

*corresponding authors: emily.derbyshire@duke.edu

Keywords: Plasmodium, malaria, vivax, hypnozoites, tubovesicular network, AQP3 


\begin{abstract}
The apicomplexan Plasmodium parasites replicate in the liver before causing malaria. $P$. vivax can also persist in the liver as dormant hypnozoites and cause relapses upon activation. The host water and solute channel aquaporin-3 (AQP3) has been shown to localize to the parasitophorous vacuole membrane (PVM) of $P$. vivax hypnozoites and liver schizonts, along with other Plasmodium species and stages. In this study, we use high-resolution microscopy to characterize temporal changes of the tubovesicular network (TVN), a PVM-derived network within the host cytosol, during $P$. vivax liver-stage infection. We demonstrate an unexpected role for the TVN in hypnozoites and reveal AQP3 associates with TVN-derived vesicles and extended membrane features. We further show AQP3 recruitment to Toxoplasma gondii. Our results highlight dynamic host-parasite interactions that occur in both dormant and replicating liver-stage $P$. vivax forms and implicate AQP3 function during this time. Together, these findings enhance our understanding of AQP3 in apicomplexan infection.
\end{abstract}

\title{
Introduction
}

Apicomplexans constitute a large group of parasitic protozoans that cause diseases, including the five species of Plasmodium that cause human malaria. Human infection by Plasmodium begins when a female Anopheles mosquito deposits sporozoites into the bloodstream which then migrate to the liver. Once in a hepatocyte, sporozoites transform and rapidly replicate to yield tens of thousands of merozoites from a single schizont (Ejigiri and Sinnis, 2009; Frischknecht et al., 2004; Ploemen et al., 2009; Prudêncio et al., 2006). This stage is asymptomatic but a prerequisite to the infection of red blood cells (RBCs) that leads to disease. Many species of Plasmodium, including $P$. falciparum and $P$. berghei, follow this path, while others such as $P$. vivax can differentiate in hepatocytes to either schizonts or hypnozoites, a biologically quiescent form (Krotoski et al., 1982). Dormant hypnozoites cause recurrent blood-stage infections (relapses) upon activation, effectively sustaining repeated blood infection and furthering transmission (Krotoski et al., 1982; Mikolajczak et al., 2015). P. vivax hypnozoites are insensitive to most antimalarials except 8-aminoquinolines, which are contraindicated in populations with G6PD deficiency, highlighting the need for new anti-hypnozoite agents (Howes et al., 2012; Lu and Derbyshire, 2020). Unfortunately, efforts to identify compounds capable of inhibiting hypnozoites are hampered by our limited understanding of the molecular pathways that enable parasite survival and activation during this stage.

To successfully develop and replicate within their host, Plasmodium resides within a membrane-bound compartment formed during invasion termed the parasitophorous vacuolar membrane (PVM) (Meis et al., 1983). Derived from the host membrane itself, the PVM serves as the host-pathogen interface and dynamically changes to facilitate development by enabling nutrient acquisition and protecting the parasite from apoptosis (Kaushansky et al., 2013; Meireles et al., 2017; Sá E Cunha et al., 2017; Van De Sand et al., 2005; Van Dijk et al., 2005). For example, the PVM diameter can expand beyond that of the pre-invasion hepatocyte membrane diameter and can recruit both host and parasite proteins. The precise composition of this membrane remains unknown, but several studies have revealed it changes minutes to hours after liver invasion when parasite proteins, including up-regulated in infective sporozoites gene 4 (UIS4), are translocated to the PVM (Kaushansky and Kappe, 2015; Mueller et al., 2005; Prado et al., 2015; Sá E Cunha et al., 2017; Schnider et al., 2018). While fewer host proteins are known to associate with the PVM after invasion, examples of protein recruitment crucial for host defense and parasite development have been reported (Grützke et al., 2014; LaMonte et al., 2019; Niklaus et al., 2019; Posfai et al., 2020, 2018; Prado et al., 2015; Raphemot et al., 2019; Thieleke-Matos 
et al., 2016; Wacker et al., 2017). In particular, we have demonstrated that the host water and solute channel aquaporin 3 (AQP3) is upregulated after $P$. berghei infection of liver cells, is critical for parasite development, and associates with the PVM (Posfai et al., 2018). Importantly, we have established the recruitment of AQP3 in multiple Plasmodium species and stages including liverstage $P$. berghei, blood-stage $P$. falciparum, blood-stage $P$. vivax, and liver-stage $P$. vivax (schizonts and hypnozoites) (Posfai et al., 2020, 2018). While the reduced intrahepatic $P$. berghei size observed with genetic or chemical inhibition of AQP3 supports an essential role in Plasmodium development, its molecular function during infection has yet to be resolved.

A key component of the host-parasite interactions in the Plasmodium liver stage is the physical alteration of the PVM. Several elegant studies have shown that the PVM can extend into the host hepatocyte with a highly dynamic membranous system, the tubovesicular network (TVN) (Agop-Nersesian et al., 2018, 2017; Grützke et al., 2014; Niklaus et al., 2019). In the $P$. berghei liver-stage, this system consists of elongated membrane clusters, tubules, and vesicles that move to and from the PVM (Grützke et al., 2014). The TVN expansion into the host cytosol is proposed to aid in nutrient acquisition and immune evasion, imparting key survival strategies to the parasite (Agop-Nersesian et al., 2018, 2017; Grützke et al., 2014; Niklaus et al., 2019). While UIS4 is an established parasite-derived $P$. berghei TVN marker, the only host proteins reported to associate with TVN features are LC3, LAMP1, p62 and CD63 (Agop-Nersesian et al., 2018, 2017; Grützke et al., 2014; Niklaus et al., 2019). There has been no evidence of a protein responsible for nutrient uptake to be associated with the TVN. In the blood-stage, the TVN is complemented by an unusual protein trafficking system that involves long-known parasite-derived membranous structures in the RBC cytosol, termed Maurer's clefts in $P$. falciparum and Schüffner's dots in $P$. vivax (Akinyi et al., 2012; Alkawa et al., 1975; Mundwiler-Pachlatko and Beck, 2013; Sakaguchi et al., 2016; Schüffner, 1899; Spycher et al., 2006; Tamez et al., 2008; Tokumasu et al., 2014; Wickert et al., 2004; Wickert and Krohne, 2007). Notably, these structures have not been found in infected hepatocytes. Additionally, the TVN has not yet been reported in liver-stage $P$. vivax, likely due to the technical difficulties associated with studying parasites during this stage.

Recent advances in $P$. vivax model systems that can support liver-stage schizont and hypnozoite development have been instrumental to setting the stage for anti-hypnozoite drug screens and aiding molecular studies to understand parasite biology (Antonova-Koch et al., 2018; Gural et al., 2018; Maher et al., 2020; March et al., 2013; Mikolajczak et al., 2015; Roth et al., 2018). Here, we analyzed $P$. vivax-infected primary human hepatocytes $(\mathrm{PHH})$ to discover and characterize TVN features throughout infection (Roth et al., 2018). Interestingly, we observe that TVN features are more abundant in dormant hypnozoites than in replicating schizonts. We also found that AQP3 co-localizes with TVN vesicles and extended membrane clusters at certain time points in $P$. vivax hypnozoites as well as liver-stage $P$. vivax and $P$. berghei schizonts, implicating a role in solute transport or evasion of host defense mechanisms. Additionally, we observe AQP3 localization to the apicomplexan parasite Toxoplasma gondii and upregulation of $A Q P 3$ expression after parasite infection of hepatocytes. Together, our work suggests a possibly conserved role for AQP3 in apicomplexans to aid in development through nutrient acquisition or protection from host defenses.

\section{Results}

\section{P. vivax TVN characterization}

The $P$. berghei liver-stage TVN is known to be important for nutrient acquisition and immune evasion, but this network has not yet been characterized in the $P$. vivax liver-stages. $P$. 
berghei requires approximately 2 days to mature from a sporozoite to a schizont in the liver (Figure 1 -figure supplement 1), while $P$. vivax schizont maturation occurs over 8-10 days in vitro. $P$. vivax also has an alternative morphological and physiological route that arrests development to create a quiescent hypnozoite that can activate days or months after infection (Figure 1A). To examine the possibility of TVN formation in $P$. vivax schizonts or hypnozoites, we used highresolution confocal microscopy to obtain images of $P$. vivax-infected $\mathrm{PHH} 2-10$ days postinfection (dpi). Parasites were stained with a recombinant anti-Plasmodium UIS4 (rUIS4) antibody (Schafer et al., 2018) to visualize the PVM/TVN and DAPI to evaluate number of parasite nuclei. This analysis showed that TVN features were present throughout $\mathrm{PHH}$ infection with $P$. vivax, with a higher percentage of exoerythrocytic forms (EEFs) positive for TVN features when observed on days 5 and 6 post-infection (Figure 1B). While the schizont and hypnozoite populations are of similar sizes and are difficult to distinguish up to 5-6 dpi, our analysis suggested TVN features were more abundant in smaller sized parasites when observed at 8 dpi. To explore this observation, we performed two classifications. First, we measured the area of each EEF and divided them into one of two categories: not exhibiting TVN features or exhibiting features at $8 \mathrm{dpi}$ (Figure 1C). This analysis revealed that the vast majority of $P$. vivax EEFs displaying TVN features were relatively small at 8 dpi (Figure 1D). Second, to definitively characterize EEF populations as hypnozoites and schizonts, we quantified the number of parasite nuclei and growth area of each EEF when imaged at high resolution (63x magnification). A uninucleated parasites indicates a non-replicating dormant hypnozoite, while a multinucleated parasite indicates a schizont or activated hypnozoite, as either would be actively replicating (Mikolajczak et al., 2015). We observed all multinucleated EEFs as having an area $\geq 75 \mathrm{~cm}^{2}$, thus we established a strict size cutoff for hypnozoites as having a growth area $<60{\mu \mathrm{m}^{2}}^{2}$ (Figure 1-figure supplement 2A) and for schizonts as having a growth area above $>200 \mu \mathrm{m}^{2}$ (Figure 1 -figure supplement $2 \mathrm{~B}$ ). While few net 8 dpi EEFs were noted with a growth area between $60 \mu \mathrm{m}^{2}$ and $200 \mu \mathrm{m}^{2}$, these forms were excluded from classification of hypnozoite or schizont to ensure no ambiguity between the two forms. This more precise analysis of the small parasite population revealed the vast majority of the EEFs displaying TVN features were classified as hypnozoites. Using these metrics, we found that $32 \%$ of hypnozoites had one or more TVN features at $8 \mathrm{dpi}$ (Figure 1E), while no schizonts displayed features among the 529 EEFs analyzed on this dpi.

Through our analysis, we observed that most $P$. vivax liver-stage EEFs displaying a TVN had multiple features (2-3). To further examine these features and the relationship with $P$. vivax hypnozoites and schizonts, we classified $P$. vivax TVN features as extended membrane clusters (mem. cluster), tubules, and vesicles (Figure 2A), similar to a previous liver-stage TVN $P$. berghei study (Grützke et al., 2014). This analysis revealed that TVN-derived vesicles in the host cytosol were most prominent in cells containing hypnozoites at $8 \mathrm{dpi}$, present within $30 \%$ of the population (Figure 2B). Extended membrane clusters and tubules were present in $\sim 15 \%$ of the hypnozoite population. Throughout infection, the $P$. vivax TVN includes multiple features, where the majority of EEFs displaying extended membrane clusters or tubules also displayed TVN-derived vesicles (Figure 2-figure supplement 1A). These TVN-derived vesicles are sometimes seen close to the EEF, but also can be found at distal parts of the cell.

We hypothesized that TVN features may vary depending on the parasite's developmental stage. Therefore, we took a longitudinal approach to our analysis and evaluated TVN features every day of a 10-day infection of PHH with $P$. vivax. Since hypnozoites and schizonts cannot be reliably distinguished by size before 5-6 dpi, EEFs were not separated into hypnozoites or schizonts. We discovered variation in the prevalence of different TVN features throughout infection (Figure 2C). In $P$. berghei, TVN features are more prevalent at early times of hepatocyte infection, appearing within 30 min and being most abundant between 6-24 hours post-infection (hpi) (Grützke et al., 2014). Here, we observe that early $P$. vivax EEF stages (2-3 dpi), display 
few TVN features, but a marked increase in extended membrane clusters and TVN-derived vesicles occurs from 2 to $6 \mathrm{dpi}$. Based on this analysis, 3-5 and 6-8 dpi are particularly dynamic times where the greatest changes in TVN abundance and composition were observed. For example, a remarkable decrease in extended membrane clusters is observed between 6 to $8 \mathrm{dpi}$, such that less are present at $8 \mathrm{dpi}$ than at the early time points (i.e. 2 dpi). While a decrease in TVN-derived vesicles was also observed between 6 to $8 \mathrm{dpi}$, the prevalence of this feature within cells remained high compared to that of extended membrane clusters. TVN-derived vesicles were maintained in $>20 \%$ of EEFs throughout the course of infection. Relatively less change in tubule abundance was observed throughout intrahepatic $P$. vivax development. These tubules vary in length and, interestingly, we have observed some instances of tubule association with the host nuclei (Figure 2-figure supplement 1B). On days 8-10 post-infection, when schizonts can be distinguished from hypnozoites, we evaluated TVN features as a function of size. Though we did not observe TVN features in schizonts at $8 \mathrm{dpi}$, we observed a TVN positive population of schizonts at days 9 and 10 (Figure 2-figure supplement 2A). Hypnozoites also displayed TVN features at 9 and $10 \mathrm{dpi}$ (Figure 2-figure supplement 2B).

\section{AQP3 is associated with the Plasmodium TVN}

The precise compositions of the Plasmodium liver-stage PVM and TVN remain to be resolved. We recently established that host AQP3 is recruited to the PVM of $P$. berghei (Posfai et al., 2018) and $P$. vivax EEFs, including both developing schizonts and dormant hypnozoites (Posfai et al., 2020, 2018). To better understand AQP3 recruitment to these different forms, we visualized $P$. berghei-infected $\mathrm{HuH} 7$ cells and $P$. vivax-infected $\mathrm{PHH}$ after staining with DAPI, antirUIS4 and anti-human AQP3 antibodies (Posfai et al., 2020, 2018). We observed that AQP3 colocalizes to some, but not all TVN features in $P$. berghei and $P$. vivax EEFs at 2 and 8 dpi, respectively (Figure $3 \mathrm{~A}$ and Figure 3 -figure supplement 1 ). To further investigate AQP3 recruitment to the TVN, we analyzed $P$. vivax EEFs days $2-10$ post-infection (Figure 3B). At early time points (2-3 dpi), AQP3 is not observed in every P. vivax EEF (Posfai et al., 2020); however, it was highly recruited to extended membrane clusters and vesicles when present. For example, when an EEF exhibited both AQP3 and extended membrane TVN features at 2 dpi, they were observed together in every EEF examined in our analysis. Likewise, AQP3 was highly correlated with TVN-derived vesicles at $3 \mathrm{dpi}$. Notably, we observed no AQP3 recruitment to TVN features in EEFs that lacked protein recruitment to the PVM. Further, we found no instance of AQP3 colocalizing with $P$. vivax TVN tubules at any day post infection despite looking in multiple planes (z-stacks).

\section{AQP3 is recruited during $T$. gondii infection}

We sought to more broadly examine the role of AQP3 in apicomplexan parasites. In particular, T. gondii, the causative agent of toxoplasmosis, is considered a model apicomplexan organism due to its ability to infect almost any multinucleate cell with a high infection rate (Black and Boothroyd, 2000). This intracellular parasite also resides in a parasitophorous vacuole which creates a barrier between the host cytoplasm and the growing parasite. We evaluated the recruitment and gene expression of AQP3 after $T$. gondii Pru A7 infection of various host cells. In T. gondii-infected $\mathrm{HuH} 7$ cells, we observed recruitment of AQP3 to parasites at both 24 hpi and $48 \mathrm{hpi}$ (Figure 4A). At $24 \mathrm{hpi}$, AQP3 is observed in 10\% of infected cells and is associated with the parasite plasma membrane (PPM). By $48 \mathrm{hpi}$, AQP3 recruitment is observed in $>50 \%$ of infected $\mathrm{HuH} 7$ cells and it is most commonly observed surrounding tachyzoite rosettes. At this time AQP3 is also observed at the residual body of some but not all rosettes (Figure 4-figure supplement 1). In addition to T. gondii Pru A7-infected HuH7 cells, AQP3 recruitment is observed in T. gondii Pru A7-infected Vero cells at 24 and 48 hpi (Figure 4-figure supplement 2A) and in 
T. gondii $\mathrm{RH}$-infected HuH7 cells at 48 hpi (Figure 4-figure supplement 2B). T. gondii Pru A7 is a type II strain while $T$. gondii $\mathrm{RH}$ is type I. These results demonstrate AQP3 recruitment to various T. gondii strains and in different mammalian cell lines.

Based on the relative increase in AQP3 fluorescence signal observed in our confocal images of uninfected versus $T$. gondii-infected cells, we predicted gene expression was upregulated after infection, similar to the upregulation observed after $P$. berghei infection of hepatocytes (Posfai et al., 2018). To test this, we evaluated AQP3 levels in HuH7 after infection with $T$. gondii Pru A7. In these experiments, we typically observe $\sim 50 \%$ parasite infection rates, therefore cells were not sorted but analyzed as a mixed population of infected and uninfected cells. We observed that $A Q P 3$ is upregulated at 24 (1.9-fold change, $p=0.0034)$ and 48 (2.5-fold change, $p=0.0003$ ) hpi when compared to uninfected cells (Figure 4B). We also observed an increase in AQP3 expression in Vero cells infected with $T$. gondii Pru A7 (Figure 4-figure supplement 2C) and HuH7 cells infected with T. gondii $\mathrm{RH}$ (Figure 4-figure supplement 2D), demonstrating the phenotype in both type I and II strains, and with various host cells.

To examine the importance of AQP3 in T. gondii infection, we employed a chemical biology approach and examined parasite growth in the presence of the known AQP3-inhibitor auphen (Martins et al., 2012). To complete this study, we first optimized the infection of HuH7 cells by a T. gondii Me49-luciferase strain (Pernas et al., 2014) in 384-well microplates for highthroughput screening (Z-factor=0.82). In this assay, compounds are added at $0 \mathrm{hpi}$ and then parasite load concurrent with host viability is assessed at $48 \mathrm{hpi}$. Using this method, we observed that auphen inhibits $T$. gondii Me49 parasite load in $\mathrm{HuH7}$ cells with an $\mathrm{EC}_{50}$ value of $2.3 \pm 0.61$ $\mu \mathrm{M}$. At auphen concentrations above $10 \mu \mathrm{M}$, the near-complete inhibition of parasite load $(100 \%$ reduction) was observed (Figure $5 \mathrm{~A}$ ). We also examined the change in $T$. gondii Pru A7 rosette size in $\mathrm{HuH} 7$ cells as a function of auphen concentration (Figure $5 \mathrm{~B}$ ). This analysis showed that auphen reduces rosette size with an $\mathrm{EC}_{50}$ value of $1.8 \pm 0.47 \mu \mathrm{M}$. At high auphen concentrations $(>10 \mu \mathrm{M})$, rosette size decreased (Figure $5 \mathrm{C}$ ) by $>90 \%$ when assessed at $48 \mathrm{hpi}$. No change was observed in the $T$. gondii Pru A7 infection rate in the presence of auphen (50 or $100 \mu \mathrm{M})$ when compared to DMSO at 24 hpi (Figure 5-figure supplement 1A). A similar auphen potency was determined for reduction of $T$. gondii Pru A7 parasite size in Vero cells $\left(E_{50}=1.8 \pm 0.14 \mu \mathrm{M}\right)$. Additionally, we determined that $T$. gondii Pru A7 rosette size in Vero cells was reduced $71 \%$ in the presence of $10 \mu \mathrm{M}$ auphen (Figure 5-figure supplement 1B). Our observed auphen inhibition of $T$. gondii is similar to the potency previously reported against various Plasmodium species and stages (Figure 5D).

\section{Discussion}

$P$. vivax remains a critical hurdle to malaria eradication efforts due in large part to the parasite's ability to persist in the liver and lead to relapses in blood infections. During the liver stage, the parasites can mature into schizonts after invasion or delay replication as a seemingly arrested form termed a hypnozoite, which can unpredictively activate. Processes that drive invasion, maturation and development during this time are considered elusive, yet much less is understood about contributing factors that enable the survival of dormant hypnozoites. Our study provides insights into molecular processes occurring during this liver stage by highlighting the role of the TVN in both $P$. vivax schizonts and hypnozoites. Previously well-characterized in liver-stage $P$. berghei schizonts, the TVN is known to be critical for nutrient acquisition and evasion of the host immune responses (Agop-Nersesian et al., 2018, 2017; Thieleke-Matos et al., 2016). 
Our study provides the first description of the TVN in $P$. vivax EEFs. Interestingly, we observed differences in the utilization of this network throughout infection between $P$. vivax and $P$. berghei. Both Plasmodium species exhibit a similar sequence of developmental events that lead to schizonts, which includes invasion, morphological changes, replication, merozoite formation, and parasite release, though at varying time scales. Based on a previous report, $P$. berghei exhibits greater TVN activity before replication (Grützke et al., 2014), at early time points, yet we observe this network is present throughout a 10-day $P$. vivax time course. The rise in TVN activity between 2-6 dpi highlights an important functional role during that time. Conversely, the decline observed in TVN features after 6 dpi likely signifies a change in the EEF development, whereby TVN function is less critical. At these later stages of development, TVN-derived vesicles are the most prominent feature. TVN vesicles are expelled into the host cytosol and Grützke and colleagues have reported that they have a relatively slower velocity compared to the other studied features (Grützke et al., 2014). One possibility for our observation of these features after others have dissipated is that they may take longer to recycle but their presence throughout liver-stage infection suggests this is unlikely due to ample time for clearance. Another possibility is that vesicles observed at 9 and $10 \mathrm{dpi}$ in schizonts may facilitate egress or further protect the parasite from clearance by shedding autophagy related proteins to these TVN-derived vesicles. A realtime evaluation of TVN characteristics would be critical for evaluating the dynamics of each feature and correlating them with developmental changes within the parasites and protection from clearance by the host. This remains difficult as the genetic tools necessary to generate transgenic $P$. vivax sporozoites that constitutively express UIS4 are not yet available. Such a tool would also be critical to assess TVN features that may have been missed due to the time points selected for microscopy analysis.

While the TVN in developing $P$. vivax EEFs was anticipated based on $P$. berghei studies, its presence in hypnozoites, which are considered biologically quiescent, was less expected. In fact, at later days when hypnozoites and liver-stage schizonts can be distinguished microscopically, TVN features were more prevalent in $P$. vivax hypnozoites versus schizonts. This host-pathogen activity seems counterintuitive to notions of the dormant state and suggests that many as yet unknown complex interactions occur during this time. Indeed, recent RNAsequencing studies of $P$. vivax hypnozoites have suggested metabolic activity occurs at this time (Gural et al., 2018). In regard to the TVN, one explanation for these features is that they prime the dormant EEF for activation, essentially preparing the parasite for nuclear division. We observe a significant decrease in hypnozoites from day $6(67 \%)$ to day $10(19 \%)$, suggesting activation, or alternatively host clearance, may occur during this time. Although hypnozoite activation is an enticing hypothesis for TVN activity, the lack of knowledge about this process makes it difficult to prove. It is also possible that the EEFs displaying these features are able to evade the host immune response, something that may be further explored with future studies evaluating the association of host immune response proteins to the TVN present in hypnozoites.

We observed host AQP3, a protein channel that is permeable to water, glycerol and other small solutes, associates with the TVN in both $P$. berghei and $P$. vivax EEFs. Interestingly, various host aquaporins have been implicated in apicomplexan infection. Previous studies have shown Cryptosporidium parvum invasion is aided by aquaporin 1 and localizes to the host-parasite interface (Chen et al., 2005). Further, an RNA-seq study demonstrated the upregulation of AQP3 and AQP4 in mice with chronic-infection of T. gondii (Pittman et al., 2014). The present study adds to this possible association by demonstrating an increase in AQP3 levels after $T$. gondii infection of hepatocytes and a reduced size phenotype for T. gondii in auphen-treated $\mathrm{HuH} 7 \mathrm{cells}$, similar to that previously observed with $P$. berghei (Posfai et al., 2018). Thus, host aquaporins may represent a class of host proteins with significance to apicomplexan infection. This function may be complementary to that of apicomplexan aquaporins, which can have essential functions 
in transporting water and small solutes (Hansen et al., 2002; Miranda et al., 2010; Promeneur et al., 2018, 2007).

A well-characterized role for AQP3 in mammalian cells is in nutrient flux, a function similar to that of the TVN in liver-stage $P$. berghei EEFs (Agop-Nersesian et al., 2018). We detected AQP3 exclusively in extended membrane clusters and TVN-derived vesicles in $P$. vivax EEFs throughout infection (Figure 3B), suggesting important and coincident functions. Notably, we did not observe localization of AQP3 to all extended membrane clusters and vesicles. This partial colocalization was expected due to the transitionary state of AQP3 between 2-5 dpi, where the protein is being recruited to the $P$. vivax PVM (Posfai et al., 2020). It remains unknown if AQP3 is trafficked from the host cytosol to the PVM by TVN-derived vesicles or alternatively, AQP3 in the PVM is shed into vesicles. In support of the former hypothesis, we did not observe AQP3 localization in TVN-derived vesicles without localization to PVM concurrently. Among the host proteins known to associate with the liver-stage TVN, it has been reported that both LC3 and LAMP1 are shed from the PVM to the TVN to facilitate evasion of the host immune response (Agop-Nersesian et al., 2017; Thieleke-Matos et al., 2016). While this highlights a role for the TVN in protection from the host response, the network is also known to be important for nutrient acquisition. Previous reports have shown that host-derived endosomes and lysosomes associate with the TVN, presumably releasing their degraded contents to the network where they can then potentially be imported as nutrients (Agop-Nersesian et al., 2017; Grützke et al., 2014). AQP3 could therefore facilitate this nutrient acquisition. A possible function in nutrient acquisition may not be relevant to tubules, which may account for the lack of AQP3 association with that TVN feature. It also remains possible that we could not detect AQP3 association to TVN tubules due to their highly dynamic nature (Grützke et al., 2014) and our method of analysis, but we were confidently able to establish the connection between the host protein and TVN-extended membrane clusters and vesicles. AQP3 has also been associated with oxidative stress and the immune response, often via its ability to transport hydrogen peroxide (Hara-Chikuma et al., 2015; Miller et al., 2010; Nalle et al., 2020; Vieceli Dalla Sega et al., 2014; Wang et al., 2020). Thus, hydrogen peroxide or other as yet unidentified solutes remain plausible functions for AQP3 in the PVM/TVN of $P$. vivax schizonts and hypnozoites.

Beyond our efforts with Plasmodium, we were able to demonstrate AQP3 is recruited to T. gondii, a model apicomplexan parasite. Specifically, we observed AQP3 upregulation and recruitment to the PPM at $24 \mathrm{hpi}$ and to the PVM at 48 hpi in T. gondii Pru A7-infected hepatocytes. Interestingly, AQP3 induction was also observed in chronic $T$. gondii-infected mice (Pittman et al., 2014). At $24 \mathrm{hpi}$, we observed AQP3 recruitment to the PPM in 10\% of T. gondii-infected $\mathrm{HuH} 7$ cells. This recruitment changed by 48 hpi where AQP3 was observed in the PVM in $~ 50 \%$ of infected cells. At this time, we also observed AQP3 localization to the residual body. While not present in Plasmodium, the residual body is known to help structural components of the rosette, facilitate division, and exchange solutes in Toxoplasma (Coppens and Romano, 2018; Frénal et al., 2017; Periz et al., 2017). Therefore, the presence of AQP3 at this junction could aid in solute transport. Notably, T. gondii also displays a TVN, although this membranous structure is present between the PPM and the PVM, whereas in the Plasmodium LS, the TVN is comprised between the PVM and host cytosol. While we were unable to detect the T. gondii TVN under our experimental conditions, the recruitment of AQP3 suggests that it may aid in survival for some parasite populations.

Of note, only $\sim 50 \%$ of $T$. gondii rosettes have AQP3 recruitment by 48 hpi when compared the $100 \%$ recruitment observed with $P$. berghei EEFs. Counterintuitive to this observation, we found that the known AQP3 inhibitor auphen completely inhibited T. gondii load in $\mathrm{HuH} 7$ cells. This finding suggests that additional parasite and/or host targets exist for auphen as it inhibits all 
parasites regardless of AQP3 recruitment. Examining single-cell gene expression in cells without AQP3 recruitment may shed light on factors that can compensate for its function during parasite infection. Importantly, AQP3 gene induction, AQP3 protein recruitment and parasite sensitivity to auphen were observed in three different T. gondii strains (Pru A7, Me49 and RH), from two lineages (type I and type II), and with different host cells (HuH7 and Vero). These results point to T. gondii as a possible model for future studies investigating AQP3 function and inhibitor screening.

Taken together, we demonstrate a previously unknown role for the TVN in dormant $P$. vivax hypnozoites and highlight the possible function of AQP3 in this network in both mouse- and human-infective liver-stage Plasmodium. AQP3 association specifically with TVN-extended membrane clusters and vesicles implicates the water and solute channel in their function throughout infection. Further, our demonstration that AQP3 is also recruited to $T$. gondii suggests a broad importance of this protein to apicomplexans. Future efforts to resolve the molecular function of AQP3 throughout schizont and hypnozoite development as well as studies to map host and parasite constituents of the TVN will be critical to advancing our understanding of hostparasite interactions during this critical time of the life cycle.

\section{Materials and Methods}

\section{Lead contact and materials availability}

Further information and requests for $P$. vivax-related reagents should be directed to Benoît Witkowski (bwitkowski@pasteur-kh.org). There are restrictions on the availability of some $P$. vivax-related reagents due to inadequate methodology for the preservation and propagation of the parasites in clinical isolates. This study did not generate new unique reagents from Emily Derbyshire. Further information about auphen and imaging reagents should be directed to and will be fulfilled by the Lead Contact, Emily Derbyshire (emily.derbyshire@duke.edu).

\section{P. vivax infections of primary human hepatocytes $(\mathrm{PHH})$}

$P$. vivax infections were completed as previously described (Roth et al., 2018). Blood samples were collected from symptomatic $P$. vivax patients at local health facilities in Mondulkiri province (eastern Cambodia) from 2018-2019. Clinical isolate collection and research procedures were reviewed and approved by the Cambodian National Ethics Committee for Health Research (approval number: \#101NECHR\& \#273NECHR). Patients presenting signs of severe malaria, infected with non-vivax malaria parasites, under 5 years of age, or who were pregnant or lactating were excluded from the collection. Following informed consent from eligible study participants, venous blood samples were collected by venipuncture into heparin-containing tubes (Beckton Dickinson, Cat\# 367886). Venous blood was pelleted and serum was replaced with naïve human serum (Interstate Blood bank, Inc.) before feeding to An. dirus mosquitoes using glass bell insect feeders held at $37^{\circ} \mathrm{C}$. Following a $P$. vivax gametocyte-containing bloodmeal, An. dirus mosquitoes were maintained on a natural light cycle and $10 \%$ sucrose in water. Mosquitoes found positive for $P$. vivax oocysts at six days post-feeding were transported to the IPC facility in Phnom Penh, Cambodia where salivary glands were aseptically dissected into RPMI without sodium bicarbonate (Gibco, Cat\# 61870-010) on day 16-21 post-feeding. Cryopreserved PHH were thawed into InVitroGro ${ }^{\mathrm{TM}}$ CP Medium (BiolVT) including a 1x antibiotic mixture (PSN, Gibco and Gentamicin, Gibco) and 18,000 live cells were added to selected wells of a collagen-coated 384well plate (Grenier). Cultures were maintained in a standard tissue culture incubator at $37^{\circ} \mathrm{C}$ and $5 \% \mathrm{CO}_{2}$. Two lots of PHH were used for IFA analysis: lot BGW was obtained from a 50 -year old Caucasian male, lot UBV was obtained from a 57-year old Caucasian male. Infection of PHH was 
performed at 2 days post-seed by diluting freshly dissected sporozoites into CP media with antibiotics, adding $20 \mu \mathrm{L}$ sporozoite-media mixture to each well, and centrifugation the 384-well plate at 200 RCF for $5 \mathrm{~min}$ at room temperature. Media was exchanged with fresh CP media containing antibiotics the day after infection and every 2-3 days thereafter.

\section{Liver stage $\boldsymbol{P}$. vivax immunofluorescent (IFA) microscopy and TVN analysis}

P. vivax-infected $\mathrm{PHH}$ were fixed with $4 \%$ paraformaldehyde (ThermoFisher) for $1 \mathrm{hr}$. Fixed cells were stained with recombinant mouse anti- $P$. vivax Upregulated in Infectious Sporozoites-4 antibody (rPvUIS4, (Schafer et al., 2018)) (1:2,500) in staining buffer $(0.03 \%$ TritonX-100, $1 \%$ $(\mathrm{w} / \mathrm{v}) \mathrm{BSA}$ in PBS) overnight at $4^{\circ} \mathrm{C}$. Cells were then washed with PBS and stained with rabbit anti-mouse Alex Fluor ${ }^{\circledR}$ 488-conjugated antibody (1:1000) in staining buffer overnight at $4{ }^{\circ} \mathrm{C}$. Cells were then washed with PBS, stained with rabbit anti-HsAQP3 (AbClonal) (1:200) for $48 \mathrm{hrs}$ at $4^{\circ} \mathrm{C}$, washed again with PBS and incubated with donkey anti-rabbit Alexa Fluor $\AA$ 568conjugated antibody (ThermoFisher) (1:400). Stained cells were washed with PBS and counterstained with $0.5 \mu \mathrm{g} / \mathrm{mL}$ DAPI (ThermoFisher). Fluorescence was detected using a Zeiss 880 Airyscan inverted confocal and images were analyzed using ImageJ (Schindelin et al., 2012). Infections were evaluated as 2D and Z-stacked images to assess TVN structures present. AQP3 colocalization with TVN structures was observed throughout $P$. vivax liver-stage infection (210dpi). GraphPad Prism was used to generate quantitative figures.

\section{P. berghei infections of HuH7 cells}

HuH7 cells (kind gift from Dr. Peter Sorger) were cultured in DMEM + L-Glutamine (Gibco) supplemented with 10\% (v/v) heat-inactivated FBS (HIFBS) and $1 \%(\mathrm{v} / \mathrm{v})$ antibiotic/antimycotic (Sigma). Cultures were maintained in a standard tissue culture incubator at $37^{\circ} \mathrm{C}$ and $5 \% \mathrm{CO}_{2}$. Anopheles stephensi mosquitoes infected with luciferase-expressing $P$. berghei ANKA were purchased from NYU Langone Medical Center Insectary. HuH7 cells were seeded (2.5x105/well) into a 24-well plate with coverslips 24 hours prior to infection. Cells were infected with $~ 50,000 P$. berghei sporozoites per well that were freshly dissected from An. stephensi mosquitoes.

\section{Liver-stage $\boldsymbol{P}$. berghei IFA microscopy and TVN analysis}

P. berghei-infected $\mathrm{HuH} 7$ cells were fixed at $24 \mathrm{hpi}$ or $48 \mathrm{hpi}$ with $3 \%$ paraformaldehyde at room temperature for $15 \mathrm{~min}$. Cells were permeabilized with $0.2 \%$ TritonX for $10 \mathrm{~min}$, washed with PBS and blocked with $3 \%$ BSA for $1 \mathrm{hr}$ at room temperature. Cells were stained with goat anti-PbUIS4 (antibodies-online) (1:1000) for $1 \mathrm{hr}$ at room temperature, washed with PBS and incubated with secondary donkey anti-goat Alexa Fluro ${ }^{\circledR} 488$-conjugated antibody (ThermoFisher) $(1: 400)$. Cells were then stained sequentially with rabbit anti-AQP3 (Rockland) $(1: 100)$ overnight at $4^{\circ} \mathrm{C}$ and donkey anti-rabbit (ThermoFisher) $(1: 400)$ for $1 \mathrm{hr}$ at room temperature. Lastly, cells were stained with DAPI. Fluorescence was detected using a Zeiss Axio Observer Widefield Fluorescence Microscope and images were analyzed using ImageJ.

\section{T. gondii infections of host cells}

Vero (ATCC) and HeLa (ATCC) cells were cultured in DMEM with L-glutamine (Gibco) supplemented with $10 \%(\mathrm{v} / \mathrm{v})$ HI-FBS and $1 \%(\mathrm{v} / \mathrm{v})$ antibiotic/antimycotic (Sigma) in a standard tissue culture incubator at $37^{\circ} \mathrm{C}$ with $5 \% \mathrm{CO}_{2}$. T. gondii tachyzoites of the type II strain Prugniaud (Pru) A7 (a kind gift from Dr. Jörn Coers) and luciferase-expressing ME49 (a kind gift from Dr. John Boothroyd) were propagated in Vero or HeLa cells. Type I mCherry-expressing RH T. gondii (a kind gift from Dr. Laura Knolls) were propagated in HeLa cells. Vero or HeLa cells infected with 
parasites were scraped, and host cells were lysed by passing them through a 25-gauge needle six times to release tachyzoites. For microscopy, Vero cells $\left(2.5 \times 10^{5}\right)$ were seeded on coverslips in 24-wells plates and infected with $T$. gondii with a multiplicity of infection (MOI) of 5.

\section{T. gondii IFA microscopy and analysis}

T. gondii Pru A7-infected HuH7 cells were fixed at $24 \mathrm{hpi}$ or $48 \mathrm{hpi}$ with $4 \%$ paraformaldehyde for $15 \mathrm{~min}$ at room temperature. Cells were washed with PBS and blocked with $3 \%$ BSA for $1 \mathrm{hr}$ at room temperature. Cells were incubated with rabbit anti-HsAQP3 (AbClonal) (1:200) for 72 hours at $4{ }^{\circ} \mathrm{C}$, washed with PBS and incubated with donkey anti-goat Alexa Fluor® 568 (ThermoFisher) $(1: 400)$ for $1 \mathrm{hr}$ at room temperature. After washing with PBS, cells were stained with DAPI $(0.5$ $\mu \mathrm{g} / \mathrm{mL}$ ) and washed once more. ProLong® Gold Antifade (ThermoFisher) was added to stained wells for preservation of fluorescence. Fluorescence was detected using a Zeiss 880 Airyscan inverted confocal microscope and images were analyzed using ImageJ. A minimum of three independent experiments, 50 parasites/experiment, were completed for all localization studies.

T. gondii parasite size in the presence of auphen was assessed with high-content imaging in Vero cells. Auphen $(20-0.01 \mu \mathrm{M})$ was added to T. gondii Pru A7-infected Vero cells before infection. Vero cells $(4,000$ cells/ wells) were infected with a $\mathrm{MOI}$ of 0.375 in a 384-well plate. All wells were at a final volume of $30 \mathrm{uL}$ and a final concentration of $1 \%$ DMSO. The number of rosettes in every DMSO- and auphen-treated well was quantified 48 hpi. T. gondii parasite size was assessed using the GFP reporter and DAPI was used to assess Vero cell numbers using the Cellomics ArrayScan (ThermoFisher) high-content imaging system. $\mathrm{EC}_{50}$ values were determined by fitting data to a standard dose-response equation (GraphPad Prism). Two independent experiments were performed with 5-10 wells/condition in each experiment.

\section{qRT-PCR}

T. gondii-infected $\mathrm{HuH7}$ cells were harvested from 6-well plates 2 dpi and resuspended into lysis buffer. RNA was extracted using the Quick-RNA miniprep (Zymo), and cDNA was synthesized using GoScript ${ }^{\mathrm{TM}}$ Reverse Transcriptase System (Promega) according to the manufacturer's instructions. The qRT-PCR analysis was performed with the oligonucleotide primers listed in STAR Methods and SYBR Green I Master reagents with a LightCycler® 480 Instrument II (Roche Diagnostics). The final volume was $5 \mu \mathrm{L}$ in a 384-well plate. HsAQP3 levels were normalized to $H$. sapiens $18 \mathrm{~S}$ in $T$. gondii-infected and uninfected cells. Each sample was analyzed in triplicate from 3 independent experiments.

\section{Toxoplasma parasite inhibition assays}

$\mathrm{HuH} 7$ cells $(7,000 /$ well) were seeded in 384-well plates in the presence or absence of auphen $(20-0.01 \mu \mathrm{M})$ in triplicate before infection with luciferase-expressing $T$. gondii-Me49 tachyzoites (6,000 parasites/well). After $45 \mathrm{hrs}$, HuH7 viability and T. gondii parasite load were assessed using the protocol described above. The relative signal intensity of each well was normalized to the negative control (1\% DMSO). The positive control was pyrimethamine $(10 \mu \mathrm{M})$. $\mathrm{EC}_{50}$ s were determined using GraphPad Prism and the reported values are the average determined from three independent experiments.

\section{Acknowledgments}


We thank the New York University and the University of Georgia, Athens Insectaries for providing $P$. berghei-infected mosquitoes, the Duke Microscopy Core Facility and the Duke Functional Genomics Facility. We thank Dr. Jörn Coers (Duke Medical School), Dr. Laura Knolls (UWMadison) and Dr. John Boothroyd (Stanford University) for providing T. gondii strains, Prugniaud A7 (GFP), RH (mCherry), and ME49 (luciferase), respectively. We thank the $P$. vivax patients of Mondulkiri Province, Cambodia for participating in this study. The recombinant mouse anti-PVUIS4 antibody was obtained from Noah Sather of Seattle Children's. We also thank Jack G. Ganley for thoughtful discussions on this paper and providing auphen. Work was supported by the Bill \&

Melinda Gates Foundation (OPP1023643 D.E.K.), the NIH (1DP2Al138239 to E.R.D) for laboratory support, and fellowship support by the NSF (DGE-1644868, to K.S.).

\section{References}

Agop-Nersesian C, De Niz M, Niklaus L, Prado M, Eickel N, Heussler VT. 2017. Shedding of host autophagic proteins from the parasitophorous vacuolar membrane of Plasmodium berghei. Sci Rep 7. doi:10.1038/s41598-017-02156-7

Agop-Nersesian C, Niklaus L, Wacker R, Theo Heussler V. 2018. Host cell cytosolic immune response during Plasmodium liver stage development. FEMS Microbiol Rev 42:324-334. doi:10.1093/femsre/fuy007

Akinyi S, Hanssen E, Meyer EVS, Jiang J, Korir CC, Singh B, Lapp S, Barnwell JW, Tilley L, Galinski MR. 2012. A 95kDa protein of Plasmodium vivax and P. cynomolgi visualized by three-dimensional tomography in the caveola-vesicle complexes (Schüffner's dots) of infected erythrocytes is a member of the PHIST family. Mol Microbiol 84:816-831. doi:10.1111/j.1365-2958.2012.08060.x

Alkawa M, Miller LH, Rabbege J. 1975. Caveola: vesicle complexes in the plasmalemma of erythrocytes infected by Plasmodium vivax and $\mathrm{P}$ cynomolgi. Unique structures related to Schuffner's dots. Am J Pathol 79:385-300.

Antonova-Koch Y, Meister S, Abraham M, Luth MR, Ottilie S, Lukens AK, Sakata-Kato T, Vanaerschot M, Owen E, Jado JC, Maher SP, Calla J, Plouffe D, Zhong Y, Chen K, Chaumeau V, Conway AJ, McNamara CW, Ibanez M, Gagaring K, Serrano FN, Eribez K, Taggard CM, Cheung AL, Lincoln C, Ambachew B, Rouillier M, Siegel D, Nosten F, Kyle DE, Gamo F-J, Zhou Y, Llinás M, Fidock DA, Wirth DF, Burrows J, Campo B, Winzeler EA. 2018. Open-source discovery of chemical leads for next-generation chemoprotective antimalarials. Science 362:eaat9446. doi:10.1126/science.aat9446

Black MW, Boothroyd JC. 2000. Lytic Cycle of Toxoplasma gondii. Microbiol Mol Biol Rev 64:607-623. doi:10.1128/mmbr.64.3.607-623.2000

Chen XM, O'Hara SP, Huang BQ, Splinter PL, Nelson JB, LaRusso NF. 2005. Localized glucose and water influx facilitates Cryptosporidium parvum cellular invasion by means of modulation of host-cell membrane protrusion. Proc Natl Acad Sci U S A 102:6338-6343. doi:10.1073/pnas.0408563102

Coppens I, Romano JD. 2018. Hostile intruder: Toxoplasma holds host organelles captive. PLOS Pathog 14:e1006893. doi:10.1371/journal.ppat.1006893

Ejigiri I, Sinnis P. 2009. Plasmodium sporozoite-host interactions from the dermis to the hepatocyte. Curr Opin Microbiol. doi:10.1016/j.mib.2009.06.006

Frénal K, Jacot D, Hammoudi PM, Graindorge A, MacO B, Soldati-Favre D. 2017. Myosindependent cell-cell communication controls synchronicity of division in acute and chronic stages of Toxoplasma gondii. Nat Commun 8. doi:10.1038/ncomms 15710

Frischknecht F, Baldacci P, Martin B, Zimmer C, Thiberge S, Olivo-Marin JC, Shorte SL, Ménard R. 2004. Imaging movement of malaria parasites during transmission by Anopheles mosquitoes. Cell Microbio/ 6:687-694. doi:10.1111/j.1462-5822.2004.00395.x

Grützke J, Rindte K, Goosmann C, Silvie O, Rauch C, Heuer D, Lehmann MJ, Mueller AK, 
Brinkmann V, Matuschewski K, Ingmundson A. 2014. The spatiotemporal dynamics and membranous features of the Plasmodium liver stage tubovesicular network. Traffic 15:362382. doi:10.1111/tra.12151

Gural N, Mancio-Silva L, Miller AB, Galstian A, Butty VL, Levine SS, Patrapuvich R, Desai SP, Mikolajczak SA, Kappe SHI, Fleming HE, March S, Sattabongkot J, Bhatia SN. 2018. In Vitro Culture, Drug Sensitivity, and Transcriptome of Plasmodium Vivax Hypnozoites. Cell Host Microbe 23:395-406.e4. doi:10.1016/j.chom.2018.01.002

Hansen M, Kun JFJ, Schultz JE, Beitz E. 2002. A single, bi-functional aquaglyceroporin in blood-stage Plasmodium falciparum malaria parasites. J Biol Chem 277:4874-4882. doi:10.1074/jbc.M110683200

Hara-Chikuma M, Satooka H, Watanabe S, Honda T, Miyachi Y, Watanabe T, Verkman AS. 2015. Aquaporin-3-mediated hydrogen peroxide transport is required for NF-1̂० B signalling in keratinocytes and development of psoriasis. Nat Commun 6. doi:10.1038/ncomms8454

Howes RE, Piel FB, Patil AP, Nyangiri OA, Gething PW, Dewi M, Hogg MM, Battle KE, Padilla CD, Baird JK, Hay SI. 2012. G6PD Deficiency Prevalence and Estimates of Affected Populations in Malaria Endemic Countries: A Geostatistical Model-Based Map. PLoS Med 9:e1001339. doi:10.1371/journal.pmed.1001339

Kaushansky A, Kappe SHI. 2015. Selection and refinement: 0The malaria parasite's infection and exploitation of host hepatocytes. Curr Opin Microbiol. doi:10.1016/j.mib.2015.05.013

Kaushansky A, Metzger PG, Douglass AN, Mikolajczak SA, Lakshmanan V, Kain HS, Kappe SHI. 2013. Malaria parasite liver stages render host hepatocytes susceptible to mitochondria-initiated apoptosis. Cell Death Dis 4:e762-e762. doi:10.1038/cddis.2013.286

Krotoski WA, Garnham PC, Bray RS, Krotoski DM, Killick-Kendrick R, Draper CC, Targett GA, Guy MW. 1982. Observations on early and late post-sporozoite tissue stages in primate malaria. I. Discovery of a new latent form of Plasmodium cynomolgi (the hypnozoite), and failure to detect hepatic forms within the first 24 hours after infection. Am J Trop Med Hyg 31:24-35.

LaMonte GM, Orjuela-Sanchez P, Calla J, Wang LT, Li S, Swann J, Cowell AN, Zou BY, AbdelHaleem Mohamed AM, Villa Galarce ZH, Moreno M, Tong Rios C, Vinetz JM, Lewis N, Winzeler EA. 2019. Dual RNA-seq identifies human mucosal immunity protein Mucin-13 as a hallmark of Plasmodium exoerythrocytic infection. Nat Commun 10:1-13. doi:10.1038/s41467-019-08349-0

Lu KY, Derbyshire ER. 2020. Tafenoquine: A Step toward Malaria Elimination. Biochemistry. doi:10.1021/acs.biochem.9b01105

Maher SP, Conway AJ, Roth A, Adapa SR, Cualing P, Andolina C, Hsiao J, Turgeon J, Chaumeau V, Johnson M, Palmiotti C, Singh N, Barnes SJ, Patel R, Van Grod V, Carter R, Sun HCS, Sattabongkot J, Campo B, Nosten F, Saadi WM, Adams JH, Jiang RHY, Kyle DE. 2020. An adaptable soft-mold embossing process for fabricating optically-accessible, microfeature-based culture systems and application toward liver stage antimalarial compound testing. Lab Chip 20:1124-1139. doi:10.1039/c9lc00921c

March S, Ng S, Velmurugan S, Galstian A, Shan J, Logan DJ, Carpenter AE, Thomas D, Sim BKL, Mota MM, Hoffman SL, Bhatia SN. 2013. A microscale human liver platform that supports the hepatic stages of plasmodium falciparum and vivax. Cell Host Microbe 14:104-115. doi:10.1016/j.chom.2013.06.005

Martins AP, Marrone A, Ciancetta A, Galán Cobo A, Echevarría M, Moura TF, Re N, Casini A, Soveral G. 2012. Targeting Aquaporin Function: Potent Inhibition of Aquaglyceroporin-3 by a Gold-Based Compound. PLoS One 7:e37435. doi:10.1371/journal.pone.0037435

Meireles P, Sales-Dias J, Andrade CM, Mello-Vieira J, Mancio-Silva L, Simas JP, Staines HM, Prudêncio M. 2017. GLUT1-mediated glucose uptake plays a crucial role during Plasmodium hepatic infection. Cell Microbiol 19. doi:10.1111/cmi.12646

Meis JFGM, Verhave JP, Jap PHK, Sinden RE, Meuwissen JHET. 1983. Malaria parasites - 
Discovery of the early liver form. Nature 302:424-426. doi:10.1038/302424a0

Mikolajczak SA, Vaughan AM, Kangwanrangsan N, Roobsoong W, Fishbaugher M, Yimamnuaychok N, Rezakhani N, Lakshmanan V, Singh N, Kaushansky A, Camargo N, Baldwin M, Lindner SE, Adams JH, Sattabongkot J, Kappe SHI. 2015. Plasmodium vivax liver stage development and hypnozoite persistence in human liver-chimeric mice. Cell Host Microbe 17:526-535. doi:10.1016/J.CHOM.2015.02.011

Miller EW, Dickinson BC, Chang CJ. 2010. Aquaporin-3 mediates hydrogen peroxide uptake to regulate downstream intracellular signaling. Proc Natl Acad Sci U S A. doi:10.1073/pnas.1005776107

Miranda K, Pace DA, Cintron R, Rodrigues JCF, Fang J, Smith A, Rohloff P, Coelho E, De Haas F, De Souza W, Coppens I, Sibley LD, Moreno SNJ. 2010. Characterization of a novel organelle in Toxoplasma gondii with similar composition and function to the plant vacuole. Mol Microbiol 76:1358-1375. doi:10.1111/j.1365-2958.2010.07165.x

Mueller A-K, Camargo N, Kaiser K, Andorfer C, Frevert U, Matuschewski K, Kappe SHI. 2005. Plasmodium liver stage developmental arrest by depletion of a protein at the parasite-host interface. Proc Natl Acad Sci U S A 102:3022-7. doi:10.1073/pnas.0408442102

Mundwiler-Pachlatko E, Beck HP. 2013. Maurer's clefts, the enigma of Plasmodium falciparum. Proc Natl Acad Sci U S A. doi:10.1073/pnas.1309247110

Niklaus L, Agop-Nersesian C, Schmuckli-Maurer J, Wacker R, Grünig V, Heussler VT. 2019. Deciphering host lysosome-mediated elimination of Plasmodium berghei liver stage parasites. Sci Rep 9:7967. doi:10.1038/s41598-019-44449-z

Periz J, Whitelaw J, Harding C, Gras S, Minina MIDR, Latorre-Barragan F, Lemgruber L, Reimer MA, Insall R, Heaslip A, Meissner M. 2017. Toxoplasma gondii F-actin forms an extensive filamentous network required for material exchange and parasite maturation. Elife 6. doi:10.7554/eLife.24119

Pernas L, Adomako-Ankomah Y, Shastri AJ, Ewald SE, Treeck M, Boyle JP, Boothroyd JC. 2014. Toxoplasma Effector MAF1 Mediates Recruitment of Host Mitochondria and Impacts the Host Response. PLoS Biol 12:e1001845. doi:10.1371/journal.pbio.1001845

Pittman KJ, Aliota MT, Knoll LJ. 2014. Dual transcriptional profiling of mice and Toxoplasma gondii during acute and chronic infection. BMC Genomics 15:806. doi:10.1186/1471-216415-806

Ploemen IHJ, Prudêncio M, Douradinha BG, Ramesar J, Fonager J, Van Gemert GJ, Luty AJF, Hermsen CC, Sauerwein RW, Baptista FG, Mota MM, Waters AP, Que I, Lowik CWGM, Khan SM, Janse CJ, Franke-Fayard BMD. 2009. Visualisation and quantitative analysis of the rodent malaria liver stage by real time imaging. PLoS One 4. doi:10.1371/journal.pone.0007881

Posfai D, Maher SP, Roesch C, Vantaux A, Sylvester K, Péneau J, Popovici J, Kyle DE, Witkowski B, Derbyshire ER. 2020. Plasmodium vivax Liver and Blood Stages Recruit the Druggable Host Membrane Channel Aquaporin-3. Cell Chem Biol. doi:10.1016/j.chembiol.2020.03.009

Posfai D, Sylvester K, Reddy A, Ganley JG, Wirth J, Cullen QE, Dave T, Kato N, Dave SS, Derbyshire ER. 2018. Plasmodium parasite exploits host aquaporin-3 during liver stage malaria infection. PLOS Pathog 14:e1007057. doi:10.1371/journal.ppat.1007057

Prado M, Eickel N, De Niz M, Heitmann A, Agop-Nersesian C, Wacker R, Schmuckli-Maurer J, Caldelari R, Janse CJ, Khan SM, May J, Meyer CG, Heussler VT. 2015. Long-term live imaging reveals cytosolic immune responses of host hepatocytes against plasmodium infection and parasite escape mechanisms. Autophagy 11:1561-1579. doi:10.1080/15548627.2015.1067361

Promeneur D, Liu Y, Maciel J, Agre P, King LS, Kumar N. 2007. Aquaglyceroporin PbAQP during intraerythrocytic development of the malaria parasite Plasmodium berghei. Proc Natl Acad Sci U S A 104:2211-2216. doi:10.1073/pnas.0610843104 
Promeneur D, Mlambo G, Agre P, Coppens I. 2018. Aquaglyceroporin PbAQP is required for efficient progression through the liver stage of Plasmodium infection. Sci Rep 8:655. doi:10.1038/s41598-017-18987-3

Prudêncio M, Rodriguez A, Mota MM. 2006. The silent path to thousands of merozoites: The Plasmodium liver stage. Nat Rev Microbiol. doi:10.1038/nrmicro1529

Raphemot R, Toro-Moreno M, Lu KY, Posfai D, Derbyshire ER. 2019. Discovery of Druggable Host Factors Critical to Plasmodium Liver-Stage Infection. Cell Chem Biol 26:12531262.e5. doi:10.1016/j.chembiol.2019.05.011

Roth A, Maher SP, Conway AJ, Ubalee R, Chaumeau V, Andolina C, Kaba SA, Vantaux A, Bakowski MA, Thomson-Luque R, Adapa SR, Singh N, Barnes SJ, Cooper CA, Rouillier M, McNamara CW, Mikolajczak SA, Sather N, Witkowski B, Campo B, Kappe SHI, Lanar DE, Nosten F, Davidson S, Jiang RHY, Kyle DE, Adams JH. 2018. A comprehensive model for assessment of liver stage therapies targeting Plasmodium vivax and Plasmodium falciparum. Nat Commun 9:1837. doi:10.1038/s41467-018-04221-9

Sá E Cunha C, Nyboer B, Heiss K, Sanches-Vaz M, Fontinha D, Wiedtke E, Grimm D, Przyborski JM, Mota MM, Prudêncio M, Mueller A-K. 2017. Plasmodium berghei EXP-1 interacts with host Apolipoprotein $\mathrm{H}$ during Plasmodium liver-stage development. Proc Natl Acad Sci U S A 114:E1138-E1147. doi:10.1073/pnas.1606419114

Sakaguchi M, Miyazaki N, Fujioka H, Kaneko O, Murata K. 2016. Three-dimensional analysis of morphological changes in the malaria parasite infected red blood cell by serial block-face scanning electron microscopy. J Struct Biol. doi:10.1016/j.jsb.2016.01.003

Schafer C, Dambrauskas N, Steel RW, Carbonetti S, Chuenchob V, Flannery EL, Vigdorovich V, Oliver BG, Roobsoong W, Maher SP, Kyle D, Sattabongkot J, Kappe SHI, Mikolajczak SA, Sather DN. 2018. A recombinant antibody against Plasmodium vivax UIS4 for distinguishing replicating from dormant liver stages. Malar J 17:370. doi:10.1186/s12936018-2519-7

Schindelin J, Arganda-Carreras I, Frise E, Kaynig V, Longair M, Pietzsch T, Preibisch S, Rueden C, Saalfeld S, Schmid B, Tinevez JY, White DJ, Hartenstein V, Eliceiri K, Tomancak P, Cardona A. 2012. Fiji: An open-source platform for biological-image analysis. Nat Methods. doi:10.1038/nmeth.2019

Schnider CB, Bausch-fluck D, Brühlmann F, Heussler VT, Burda P. 2018. BiolD Reveals Novel Proteins of the Plasmodium. Am Soc Microbio/ 3:1-13. doi:10.1128/mSphere.00522-17

Schüffner W. 1899. Beitrag zur kenntniss der malaria. Dtsch Arch Klin Med 428-449.

Spycher C, Rug M, Klonis N, Ferguson DJP, Cowman AF, Beck H-P, Tilley L. 2006. Genesis of and Trafficking to the Maurer's Clefts of Plasmodium falciparum-Infected Erythrocytes. Mol Cell Biol 26:4074-4085. doi:10.1128/mcb.00095-06

Tamez PA, Bhattacharjee S, Van Ooij C, Hiller NL, Llinás M, Balu B, Adams JH, Haldar K. 2008. An erythrocyte vesicle protein exported by the malaria parasite promotes tubovesicular lipid import from the host cell surface. PLoS Pathog. doi:10.1371/journal.ppat.1000118

Thieleke-Matos C, Lopes da Silva M, Cabrita-Santos L, Portal MD, Rodrigues IP, Zuzarte-Luis V, Ramalho JS, Futter CE, Mota MM, Barral DC, Seabra MC. 2016. Host cell autophagy contributes to Plasmodium liver development. Cell Microbiol 18:437-450. doi:10.1111/cmi.12524

Tokumasu F, Crivat G, Ackerman H, Hwang J, Wellems TE. 2014. Inward cholesterol gradient of the membrane system in P. falciparum-infected erythrocytes involves a dilution effect from parasite-produced lipids. Biol Open. doi:10.1242/bio.20147732

Van De Sand C, Horstmann S, Schmidt A, Sturm A, Bolte S, Krueger A, Lütgehetmann M, Pollok JM, Libert C, Heussler VT. 2005. The liver stage of Plasmodium berghei inhibits host cell apoptosis. Mol Microbio/ 58:731-742. doi:10.1111/j.1365-2958.2005.04888.x

Van Dijk MR, Douradinha B, Franke-Fayard B, Heussler V, Van Dooren MW, Van Schaijk B, 
Van Gemert GJ, Sauerwein RW, Mota MM, Waters AP, Janse CJ. 2005. Genetically attenuated P36p-deficient malarial sporozouites induce protective immunity and apoptosis of infected liver cells. Proc Natl Acad Sci U S A 102:12194-12199. doi:10.1073/pnas.0500925102

Wacker R, Eickel N, Schmuckli-Maurer J, Annoura T, Niklaus L, Khan SM, Guan J-L, Heussler VT. 2017. LC3-association with the parasitophorous vacuole membrane of Plasmodium berghei liver stages follows a noncanonical autophagy pathway. Cell Microbiol 19:e12754. doi:10.1111/cmi.12754

Wickert H, Göttler W, Krohne G, Lanzer M. 2004. Maurer's cleft organization in the cytoplasm of Plasmodium falciparum-infected erythrocytes: New insights from three-dimensional reconstruction of serial ultrathin sections. Eur J Cell Biol 83:567-582. doi:10.1078/01719335-00432

Wickert H, Krohne G. 2007. The complex morphology of Maurer's clefts: from discovery to three-dimensional reconstructions. Trends Parasitol. doi:10.1016/j.pt.2007.08.008

\section{Footnotes}

To whom correspondence should be addressed. Email: emily.derbyshire@duke.edu The authors have no competing interests. 
A

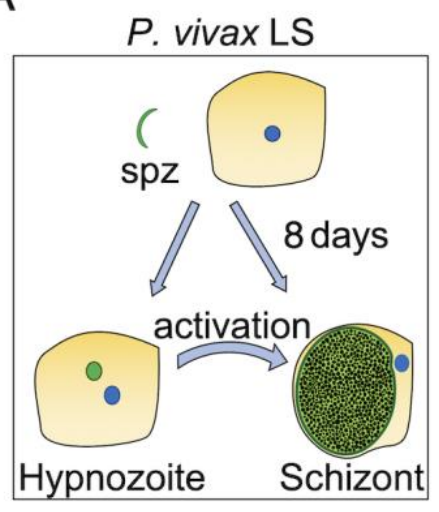

B

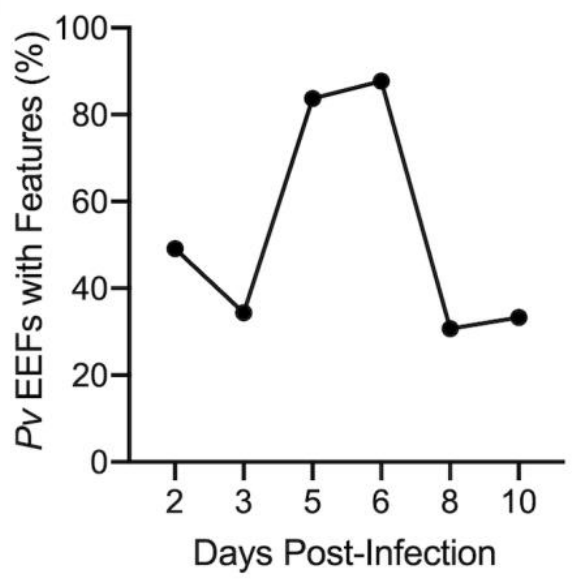

C

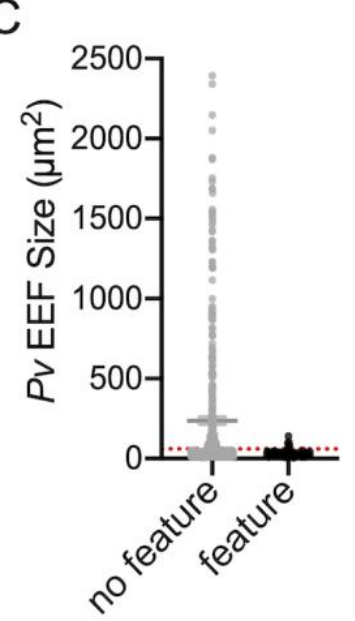

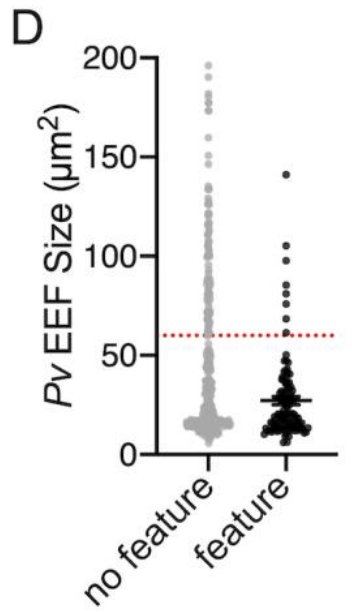

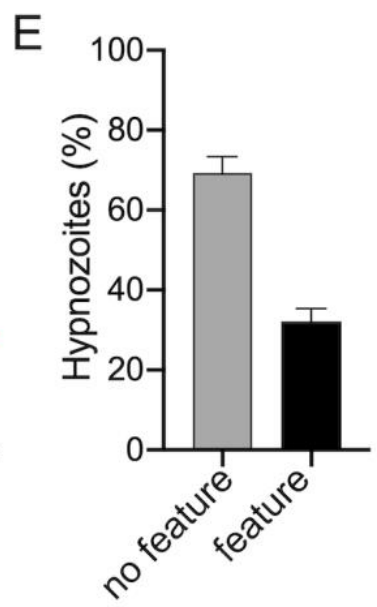

Figure 1. $P$. vivax-infected PHH contain TVN features. (A) Schematic representing $P$. vivax LS infection. (B) Percentage of $P$. vivax EEFs that contain features (black) throughout infection of PHH (2-10 dpi). (C) Relative size of P. vivax EEFs containing no TVN features (grey) or TVN feature/s (black). (D) Relative size of $P$. vivax EEFs (from C), displaying smaller scale, containing no TVN features (grey) or TVN feature/s (black). Red dotted line indicates cut off for hypnozoites $\left(60 \mu \mathrm{m}^{2}\right)$. (E) Percentage of $P$. vivax hypnozoites exhibiting no TVN features (grey bar) or TVN feature/s (black bar). (C-E) Assessed at 8 dpi (3 independent wells assessed with $>200$ EEFs, $n=2)$. 
A
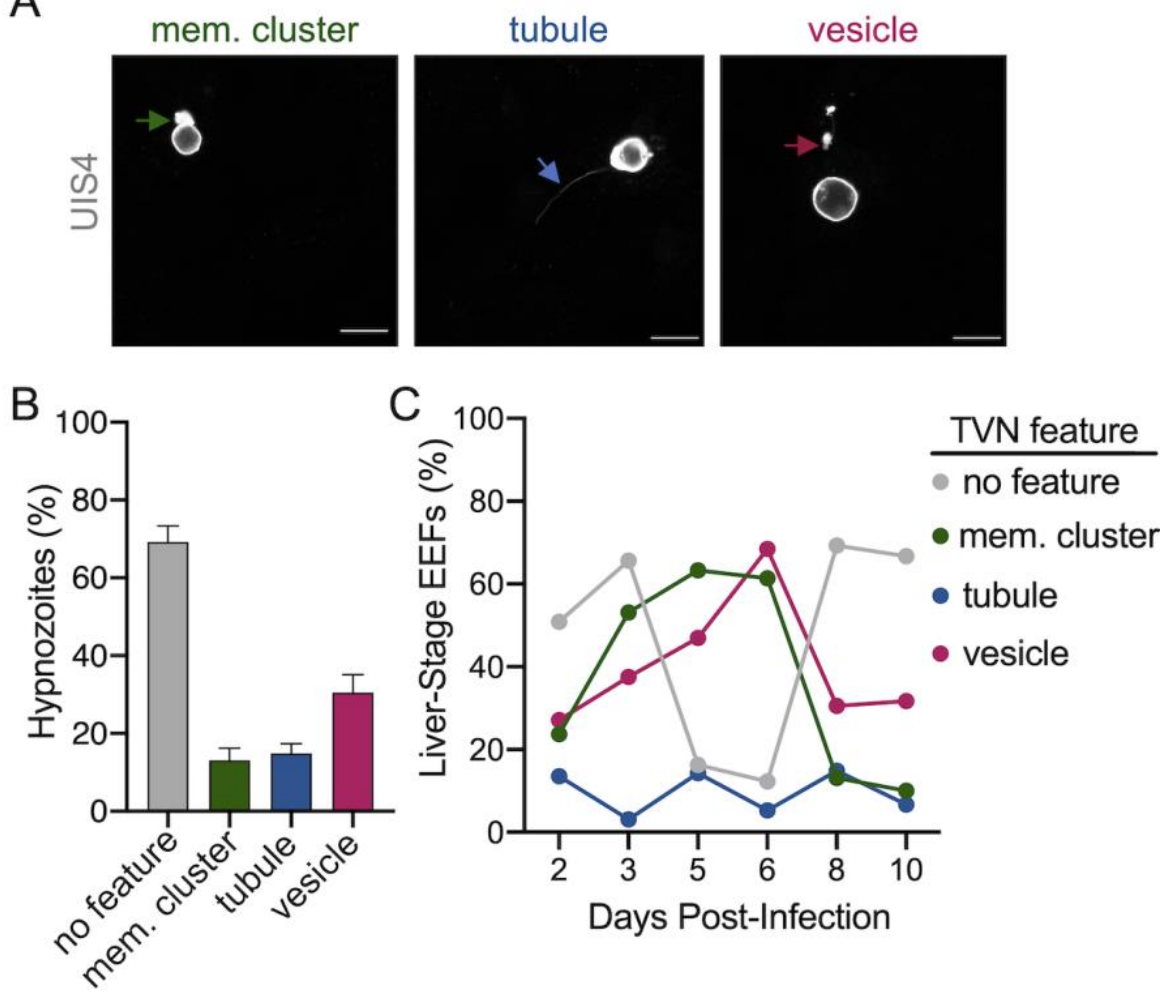

Figure 2. Characterization of $\boldsymbol{P}$. vivax TVN throughout infection. (A) Examples of TVN features including extended membrane cluster (mem. cluster, green arrow), tubule (blue arrow) and vesicles (magenta arrow) in $P$. vivax-infected PHH at 8 dpi. Cells stained with anti-PVIS4 (grey). Scale bar is $10 \mu \mathrm{m}$. (B) Characterization of $P$. vivax hypnozoites at $8 \mathrm{dpi}$ containing no feature or features. Percentage of each characteristic including extended membrane cluster (mem. cluster, green bar), tubule (blue bar), and vesicle (magenta bar) is indicated. Grey bar indicates percentage of hypnozoites with no features ( 3 independent wells assessed with >200 EEFs, $n=2$ ). (C) Percentage of total $P$. vivax EEFs with indicated TVN characteristic days $2-10$ post-infection of $\mathrm{PHH}(\mathrm{n}>30$ EEFs each day). 

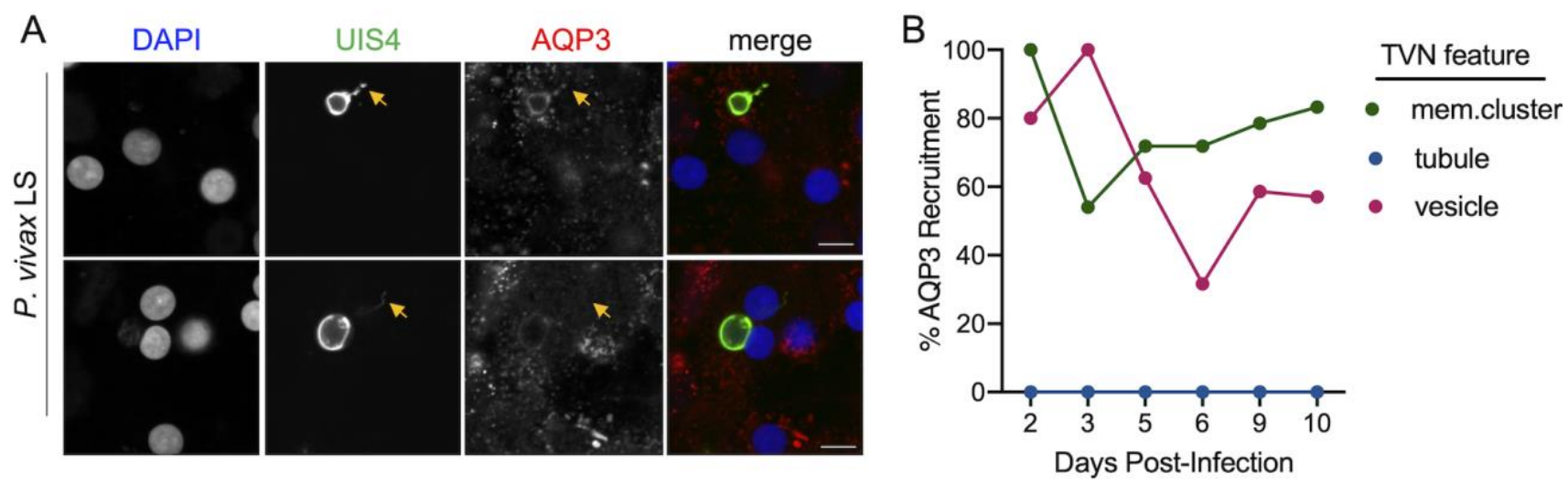

Figure 3. Localization and recruitment of AQP3 to TVN features during $P$. vivax infection. (A) Representative confocal images of $P$. vivax EEFs exhibiting TVN features, stained with DAPI (blue), anti-PVUIS4 (green), and anti-HsAQP3 (red) at $8 \mathrm{dpi}$. P. vivax-infected PHH show localization (top) and no localization (bottom) of AQP3 to specific TVN features (yellow arrows). Scale bar is $10 \mu \mathrm{m}$. (B) Relative percentage of TVN features in $P$. vivax-infected $\mathrm{PHH}$ exhibiting AQP3 recruitment (2-10 dpi, $n>30$ EEFs per dpi). 
A

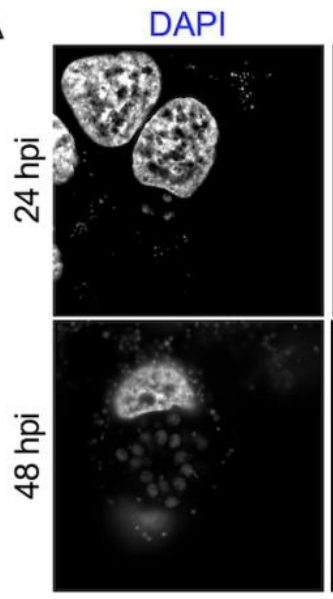

T. gondii

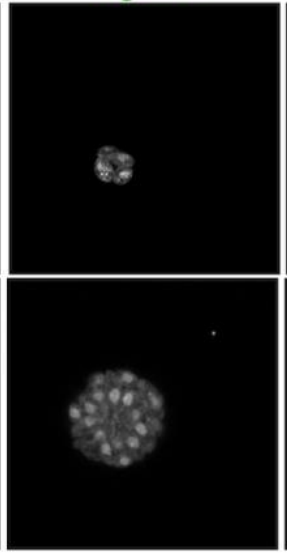

AQP3

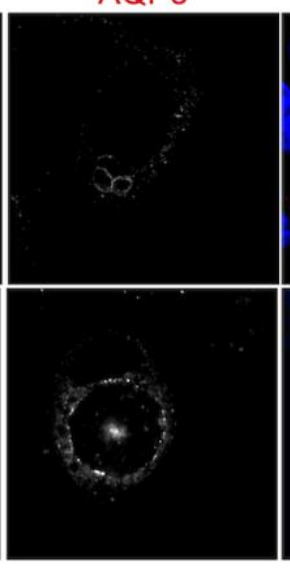

merge

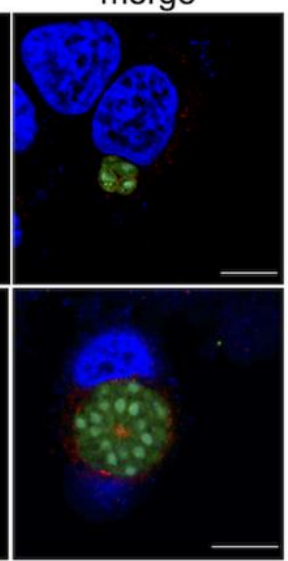

B

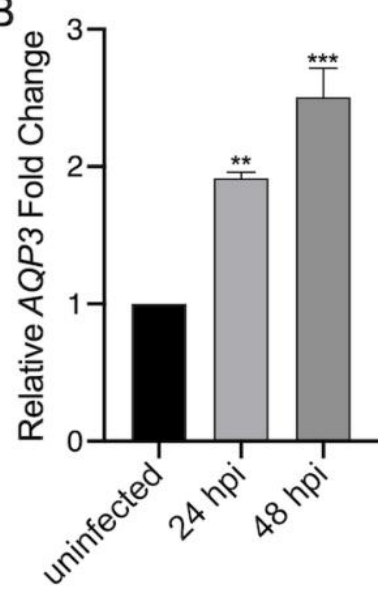

Figure 4. AQP3 recruitment and upregulation during T. gondii infection. (A) Representative confocal images of HuH7 cells infected with GFP-expressing T. gondii Pru A7 (green), stained with DAPI (blue) and anti-HsAQP3 (red) at 24 and $48 \mathrm{hpi}$. Scale bar is $10 \mu \mathrm{m}$. (B) qRT-PCR quantification of host $A Q P 3 \mathrm{mRNA}$ expression in uninfected and T. gondii Pru A7-infected $\mathrm{HuH} 7$ cells at 24 and $48 \mathrm{hpi}$. Infected cells at 24 and $48 \mathrm{hpi}$ exhibit a $1.9 \pm 0.043$-fold $(p=0.0034)$ and $2.5 \pm 0.21$-fold $(p=0.0003)$ increase, respectively, in AQP3 expression (Dunnett's multiple comparisons test; $n=3$ independent experiments). Data reported as mean $\pm S E M ; n=3$ independent experiments. ${ }^{* *} p<0.01,{ }^{* \star *} p<0.001$. 

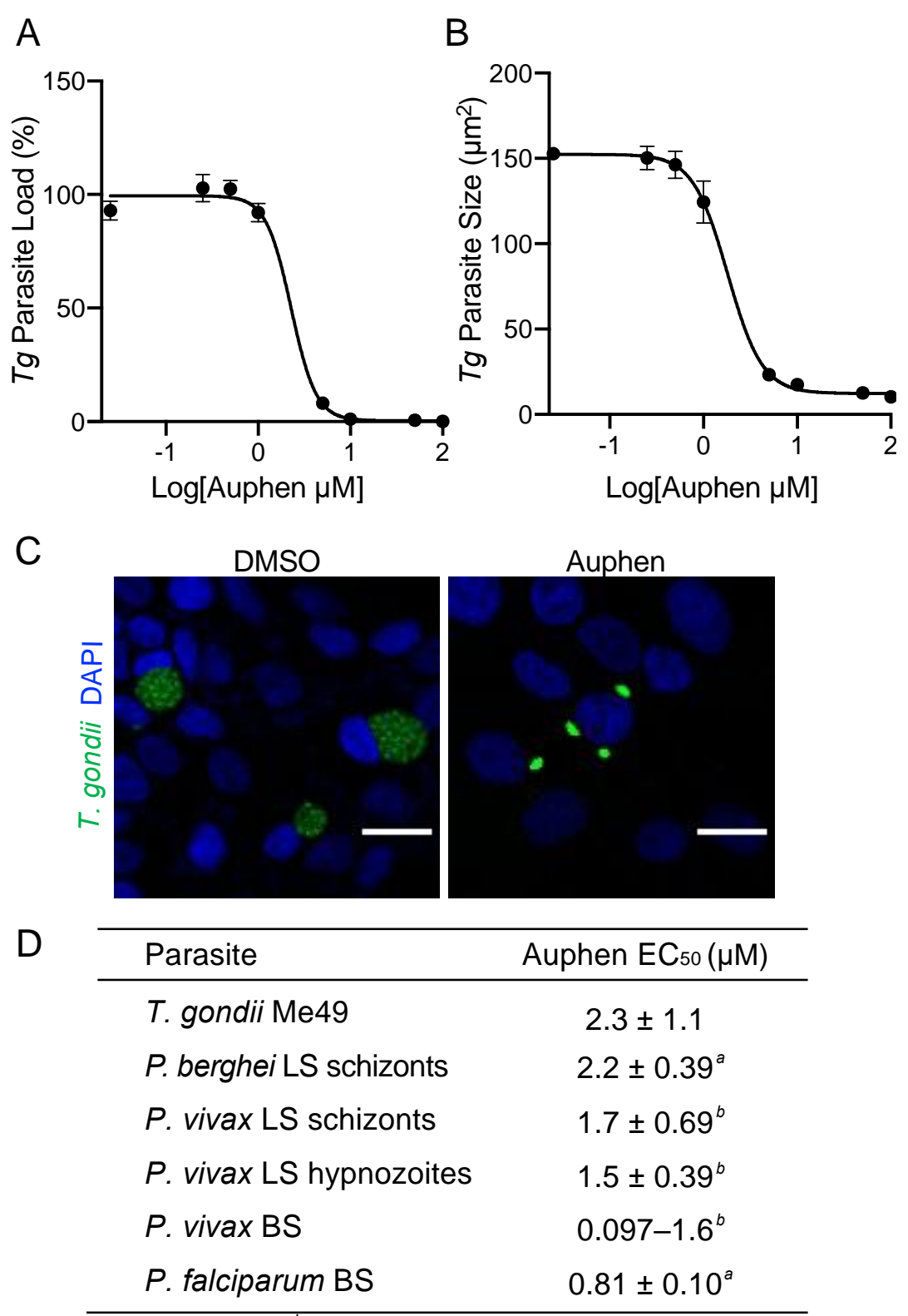

${ }^{a}$ Posfai et al. 2018; ${ }^{b}$ Posfai et al. 2020.

Figure 5. Auphen inhibition of apicomplexan parasites. (A) Auphen dose-dependent inhibition of luciferase-expressing T. gondii Me49 in $\mathrm{HuH} 7$ cells assessed at $48 \mathrm{hpi}$, evaluated with relative luminescence. Auphen $\mathrm{EC}_{50}=2.3 \pm 1.1 \mu \mathrm{M}$ (mean $\pm \mathrm{SEM} ; \mathrm{n}=3$ independent experiments). (B) Auphen dose-dependent decrease of GFP-expressing T. gondii Pru A7 rosette size in $\mathrm{HuH} 7$ cells assessed at $48 \mathrm{hpi}$, evaluated with confocal microscopy and analyzed with ImageJ. Auphen $\mathrm{EC}_{50}=1.8 \pm 0.47 \mu \mathrm{M}$ (mean $\pm \mathrm{SEM} ; \mathrm{n}=2$ independent experiments). (C) Representative confocal images (20x) of GFP-expressing T. gondii Pru A7 in HuH7 cells in the presence of DMSO (left) or $50 \mu \mathrm{M}$ auphen (right) at $48 \mathrm{hpi}$. Images display T. gondii Pru A7 (green) and DAPI (blue). Scale bar is $50 \mu \mathrm{m}$. (D) Table with $\mathrm{EC}_{50}$ values for auphen inhibition of $T$. gondii Me49 parasite load in $\mathrm{HuH} 7$ cells and various Plasmodium parasites. Parasite species and stage (LS, liver-stage; BS, blood stage) indicated for Plasmodium. Data shown as mean \pm SEM, except for $P$. vivax BS which is the range determined from various isolates. 